\title{
Adherence to guidelines for hospitalized community- acquired pneumonia over time and its impact on health outcomes and mortality
}

\section{Running Head:}

Adherence and impact of clinical guidelines for hospitalized CAP.

\section{Authors:}

Elisa Costantini, ${ }^{1,2}$ Elias Allara, ${ }^{2,3}$ Filippo Patrucco, ${ }^{4,1}$ Fabrizio Faggiano, ${ }^{2}$ Fozia Hamid,${ }^{5}$ Piero Emilio Balbo ${ }^{1}$

\author{
Affiliations: \\ ${ }^{1}$ Medical Department, Division of Respiratory Medicine, Ospedale "Maggiore della Carità", \\ Novara, Italy \\ ${ }^{2}$ Department of Translational Medicine, Università del Piemonte Orientale, Novara, Italy \\ ${ }^{3}$ School of Public Health, University of Torino, Turin, Italy \\ ${ }^{4}$ Cardiothoracic Department, Division of Respiratory Medicine, "Città della Salute e della \\ Scienza" Hospital and University of Torino, Turin, Italy \\ ${ }^{5}$ Department of Primary Care and Public Health, Imperial College London, London, U.K.
}

\section{Corresponding author:}

Filippo Patrucco

Corso Bramante 88/90, 10126 Turin, Italy

Telephone +390116336576

filippo_patrucco@hotmail.it

\section{Keywords:}

Community-acquired pneumonia, Guidelines, Adherence. 


\section{Abstract}

Background. Adherence to validated guidelines is crucial to guide management of patients hospitalized with community-acquired pneumonia (CAP). Data describing real-life management and treatment of CAP are limited.

Objective. We aimed to evaluate the adherence to guidelines over time, and to assess its impact on all-cause mortality and clinical outcomes.

Methods. We retrospectively compared two cohorts of patients admitted to hospital, throughout 2005, just after implementation of a local clinical pathway based on CAP international guidelines, and 7 years later over 2012. We included all patients with a diagnosis of pneumonia and/or related complications.

Results. 564 patients were included. Pneumonia Severity Index calculation was better documented in $2012(25.23 \%)$ compared to $2005(17.70 \% ; p=0.032)$, but adherence to guideline empirical antibiotic therapy was lower in $2012(56.70 \%)$ than in $2005(68.75 \%$; $p=0.004)$. Performance of guideline recommended urinary antigen tests was higher in 2012 and associated with $57.3 \%$ lower odds of in-hospital mortality (95\% CI $15.0 \%$ to $78.5 \%$ ) and with $65.9 \%$ lower odds of 30-day mortality (95\%CI $31.5 \%$ to $83.0 \%$ ). Adherence to empirical antibiotic therapy was associated with 2.9 days lower mean length of hospital stay (95\% CI -4.2 to -1.6 days) and with 2.0 days lower mean duration of antibiotic therapy (95\% CI -3.3 to -0.7 days).

Conclusions. Adherence to guidelines changed over time, with some effects on mortality and with an apparent reduction in length of hospital stay and duration of antibiotic therapy. Specific clinical training and hospital control policies could achieve greater adherence to guidelines and thus reduce burden on hospital services. 
Community-acquired pneumonia (CAP) is the leading cause of death for infectious disease worldwide, representing a considerable cause of morbidity and mortality, especially among hospitalized patients. [1,2] Both prevalence and hospitalization rates are particularly high in elderly and comorbid patients; $[3,4]$ for people over 65 years early readmission to hospital has also been increasing. [5] Today, if recurrences are excluded, the mean European length of hospital stay is 9.0 days and in-hospital mortality rates vary from $0 \%$ to $17.5 \%$ across countries. [6]

Given the high burden of disease and the wide spectrum of clinical severity, use of validated guidelines is crucial in guiding diagnostic and therapeutic approaches. Adherence to guidelines has been found to improve clinical outcomes, independently on contextual and patient-specific variables (such as the decision to hospitalize and the rapidity of administration of the first antibiotic dose), [6] and to reduce unnecessary hospitalizations and readmissions, length of hospital stay, costs, and mortality. [7-19]

Although adherence to guidelines varies among clinicians and is conditioned by various factors, [20] site of care, i.e. management outside or inside hospital, and correct timing of antibiotic therapy are vital factors in reducing risk of complications and mortality from CAP. [21] These aspects, as well as choice of empirical antibiotic therapy, are most critical when applying guideline indications. [6]

To comply with international guidelines, in 2005 we implemented a CAP clinical pathway (CP) based on American Thoracic Society and British Thoracic Society guidelines, and which is still largely compatible with the most updated guidelines available in 2015. [22, 23]

\section{Objectives}

We aimed to evaluate to what extent adherence to the local CAP CP in our hospital changed over time from 2005 to 2012. We also aimed to evaluate the impact of hospital-wide adherence to the local CAP CP over time on (i) in-hospital all-cause mortality, (ii) 30-day allcause mortality, (iii) length of hospital stay, and (iv) length of antibiotic therapy.

\section{MATERIAL AND METHODS}

This report was written in compliance with the STROBE Statement for observational studies. [24]

\section{Clinical pathway elaboration}

In 2004 we outlined, and in 2005 implemented, a CAP CP based on then current American Thoracic Society and British Thoracic Society guidelines. [22, 23] Our intent was to provide hospital clinicians with a concise and useful tool to manage patients with CAP. Our CAP CP comprises tools (i) to stratify patient risk and select the most appropriate setting of care, (ii) to indicate the fundamental diagnostic procedures, (iii) to select the best timing and choice of empirical antibiotic therapy (see Figure 1). 
We conducted an observational retrospective study comparing two cohorts of patients admitted throughout 2005, the year the local CAP CP was implemented, and again in 2012, seven years after its original implementation. All patients in each cohort were followed up for 30 days from admission. The study was approved by the institutional ethics committee (study number: CE 157/14).

\section{Setting}

The study was conducted in a medium size teaching hospital in North-Western Italy. The hospital has 635 beds [25] and a catchment area of approximately half a million people [26].

\section{Participants}

We included all patients $\geq 18$ years with a principal or secondary diagnosis of pneumonia and/or related complications (pleural effusion, empyema), i.e. ICD-9-CM codes 480-486, $510,511,518$, consecutively admitted to our hospital from January $1^{\text {st }}$ to December $31^{\text {st }}$ in two separate years of 2005 and 2012. We also manually inspected medical records of patients with discharge diagnosis of other pulmonary diseases, in order to capture patients with respiratory failure that could represent CAP unreported in the Hospital Discharge Records (HDR), and included them in the study whenever appropriate.

Patients admitted from the community including nursing homes were included in the study in accordance with accepted definitions of CAP in the context of the pneumonia triad. [27] Patients diagnosed with nosocomial pneumonia, including patients hospitalized for $\geq 2$ days during the prior 90 days, and immunocompromised patients were excluded. Patients with malignancies in active tratment were excluded (we included paitents with malicnancies in remission for at least 5 years). Patients whose medical records were not available were excluded too.

\section{Variables}

Variables are detailed in Figure 2.

We investigated performance indicators suggested by Infectious Diseases Society of America/American Thoracic Society (IDSA/ATS) consensus guidelines. [28]

\section{Data sources and measurements}

Information about patients was gathered through the analysis of medical records, including attached documentation and hospital discharge data, by filling out an ad-hoc dataset. The lead author and last author manually inspected each record and assessed whether or not the clinical practices were in line with those recommended by the local CAP CP. Any disagreement was resolved by consensus.

Data about 30-day mortality from admission were collected through telephone interview.

\section{Statistical methods}

We carried out descriptive analyses by testing the difference in proportions of binary mortality outcomes using $\chi^{2}$ tests and the difference in means of continuous outcomes (length of hospital stay, duration of antibiotic therapy) using Student's t tests.

To estimate the independent effects of demographic and clinical characteristics on the outcomes of interests we utilized four multivariable generalized linear models assuming a Binomial distribution of mortality outcomes and a Normal distribution of continuous outcomes (length of hospital stay and duration of antibiotic therapy), including appropriate 
link functions. Regression models included four binary parameters indicating the adherence to diagnostic procedures such as execution of blood culture and urinary antigen tests, and the adherence to therapeutic interventions such as antibiotic therapy and treatment in intensive care unit (ICU). The models also included eight potential confounders of continuous age, sex, admission from nursing home or own home, presence of comorbidities, presence of criteria for severe CAP as identified by ATS, [28] year of admission to the hospital, stay in a respiratory or non-respiratory ward, performance of mechanical ventilation (MV). For the mortality binary outcomes, all predictor effects were exponentiated to allow interpretation as odds ratio.

\section{RESULTS}

\section{Participants}

564 patients were eligible for inclusion, 243 in 2005 and 321 in 2012 (Figure 3).

Patients were admitted to 11 hospital departments: A\&E, cardiology, infectious diseases, ICU, 3 internal medicine divisions, nephrology and dialysis, geriatrics, respiratory, and thoracic surgery.

Information about patients and disease characteristics is illustrated in Tables 1 and 2.

Mean age and proportion of patients with 5 or more comorbidities were both greater in 2012 than 2005 (respectively 75.4 vs. 69.0 years; $p<0.001$; and $19.3 \%$ vs. $9.1 \%$ of patients; $p=0.001$ ), despite a significant reduction, in 2012, of proportion of severe CAP as defined by ATS criteria [28] (5.6\% of patients in 2012 vs. $10.7 \%$ in $2005 ; p=0.026$ ).

\section{Adherence to diagnostic procedures}

PSI was reported in $25.2 \%$ of patients in 2012 vs. $17.7 \%$ in 2005 ( $p=0.032$ ) (Table 3). Influenza and pneumococcal vaccination was only recorded for 1 patient.

Urinary antigen tests for S. pneumoniae and L. pneumophila were performed in a greater percentage of patients in 2012 compared to 2005 (56.4\% and $57.6 \%$ respectively in 2012 vs. $22.6 \%$ and $48.6 \%$ in 2005), although there was a significant reduction of other diagnostic tests such as blood culture, sputum Gram stain and culture, brushings and bronchial lavage in the later year.

\section{Adherence to therapeutic interventions}

Adherence to clinical pathway in choice of initial empiric antibiotic therapy was $56.7 \%$ in 2012 vs. $68.8 \%$ in 2005 ( $p=0.004$ ) (Table 4). The most common antibiotic treatment schemes in both years were levofloxacin $(16.5 \%$ in 2012 vs. $11.5 \%$ in 2005$)$, co-amoxiclav plus macrolide (14.9\% vs. $11.5 \%)$ and levofloxacin plus ceftriaxone (10.0\% vs. $12.8 \%)$ (data not shown in tables).

There was no evidence of a difference $(p=0.183)$ in the overall duration of antibiotic therapy between the two years.

$75.7 \%$ of patients admitted in 2012 and $58.9 \%$ of those admitted in 2005 appropriately received oxygen therapy $(p<0.001) .9 .35 \%$ of patients in 2012 vs. $2.06 \%$ in 2005 ( $\mathrm{p}<0.001$ ) were treated with Mechanical Ventilation (MV): in detail, $9.0 \%$ of patients in 2012 vs. $1.2 \%$ in 2005 ( $\mathrm{p}<0.001)$ were treated with non-invasive ventilation (NIV), while $1.3 \%$ of patients in 2012 vs. $1.7 \%$ in 2005 ( $\mathrm{p}=0.691)$ were treated with invasive ventilation. $2.2 \%$ of patients in 2012 vs. $1.7 \%$ in 2005 were treated in ICU ( $p=0.649)$. 


\section{In-hospital and 30-day mortality}

Mortality rates did not change significantly over time. In-hospital mortality was $13.1 \%$ in 2012 and $15.2 \%$ in $2005(p=0.468)$. Thirty-day mortality was $15.9 \%$ in 2012 and $19.6 \%$ in 2005 ( $p=0.283)$.

Adjusted regression analyses (Table 5) showed that mortality was positively associated with age and severity of pneumonia and negatively associated with performance of urinary antigen tests for S. pneumoniae and L. pneumophila. Mortality was not associated with adherence to empirical antibiotic treatment in the CAP clinical pathway.

With each one year increase in age there was an $8.1 \%$ increase in odds of in-hospital mortality (95\%CI $4.9 \%$ to $11.4 \%$ ) and a $10.5 \%$ increase in odds of 30 -days mortality $(95 \% \mathrm{CI}$ $6.8 \%$ to $14.3 \%$ ), while keeping all other predictors constant. Subjects meeting the criteria for severe CAP had $74.3 \%$ greater odds of in-hospital mortality (95\%CI $38.3 \%$ to $119.6 \%$ ) and $86.8 \%$ greater odds of 30-day mortality (95\% CI $46.2 \%$ to $138.5 \%)$ compared to subjects not meeting such criteria, while holding all other predictors constant. Performance of urinary antigen test was associated with $42.7 \%$ lower odds of in-hospital mortality (95\% CI $21.5 \%$ to $85.0 \%$ ) and $34.1 \%$ lower odds of 30 -day mortality (95\%CI $17.0 \%$ to $68.5 \%$ ) compared to subjects who were not tested for urinary antigens, while holding all other predictors constant. Association between treatment in the ICU and mortality did not reach statistical significance at the 5\% level. However, treatment in the ICU appears to result in lower odds of both inhospital mortality (OR $0.5 ; 95 \% \mathrm{CI} 0.1$ to 2.8 ) and 30-day mortality (OR 0.3 ; $95 \% \mathrm{CI} 0.1$ to 2.0).

\section{Length of hospital stay and duration of antibiotic therapy}

Mean length of hospital stay was 11.5 days in 2012 and 11.7 days in 2005 ( $p=0.831$ ). Mean duration of antibiotic therapy was 12.8 days in 2012 and 13.7 days in 2015 ( $p=0.183$ ).

Regression analyses (Table 5) showed adherence to $\mathrm{CP}$ antibiotic treatment was associated with shorter mean length of hospital stay (-2.9 days; $95 \% \mathrm{CI}-4.2$ to -1.6 days) and with shorter mean duration of antibiotic therapy ( -2.0 days; $95 \% \mathrm{CI}-3.3$ to -0.7 days). Admission from nursing home was associated with 3.2 days shorter mean length of hospital stay (95\%CI -5.2 to -1.2 days) compared with admission from home. Mean duration of antibiotic therapy was 1.1 days shorter for subjects meeting the criteria for severe CAP (95\%CI -1.7 to -0.5 days). With each one year increase in age there was a 0.1 days increase in mean duration of hospital stay ( $95 \%$ CI 0.02 to 0.1 days). Factors associated with increased length of hospital stay and duration of antibiotic therapy were: performance of MV (4.6 days; $95 \% \mathrm{CI} 1.7$ to 7.6 days; and 5.0 days; $95 \% \mathrm{CI} 2.1$ to 7.9 days respectively), admission to ICU ( 8.4 days; $95 \% \mathrm{CI}$ 3.6 to 13.3 days; and 6.2 days; $95 \%$ CI 1.2 to 11.3 days respectively), admission to respiratory ward ( 4.2 days; $95 \% \mathrm{CI} 2.2$ to 6.3 days; and 4.4 days; $95 \% \mathrm{CI} 2.4$ to 6.4 days respectively) and performance of blood cultures (2.6 days; 95\% CI 1.3 to 4.0 days; and 2.7 days; $95 \%$ CI 1.4 to 4.0 days respectively).

\section{DISCUSSION}

Our study aimed to provide an analysis of the clinical experience of a medium sized Italian teaching hospital, which is likely to be similar in other Western-European settings. 


\section{Summary of findings}

Adherence to clinical pathway. In our hospital, adherence changed over time. PSI calculation was better documented in 2012 compared to 2005, although the proportion of patients that received antibiotic therapy adherent to clinical pathway (CP) was lower in 2012.

Concerning the performance of procedures aimed at obtaining an aetiological diagnosis, urinary antigen tests for S. pneumoniae and L. pneumophila were significantly increased in 2012 compared to 2005, despite a significant reduction in most other diagnostic tests, and performed in a significantly greater proportion of patients in the respiratory ward than in other wards.

Mortality. In-hospital and 30-day mortality did not significantly change over time after adjustment for adherence to CP, although their point estimates were lower in 2012 compared to 2005. In the adjusted regression analyses, age and severity of pneumonia emerged as the only predictors associated with an increased risk of in-hospital and 30-day mortality. Patients who were tested for urinary antigens had lower odds of in-hospital and 30-day mortality. Administration of empirical antibiotic therapy adherent to $\mathrm{CP}$ did not have a significant impact on mortality from CAP.

Clinical outcomes. Mean length of hospital stay and mean length of antibiotic therapy remained almost unchanged in 2012 and 2005, although they were significantly higher among patients hospitalized in the respiratory ward compared to those treated in other departments. The administration of an empirical antibiotic therapy adherent to $\mathrm{CP}$ was associated with a reduction in length of stay and duration of antibiotic therapy. Regression analysis confirmed these observations and also provided some evidence of an increased mean length of hospital stay and duration of antibiotic therapy associated with execution of blood culture, mechanical ventilation (MV) and treatment in ICU.

\section{Comparison with the literature}

Adherence to clinical pathway. The few studies carried out so far suggest that severity scores for pneumonia are rarely used in clinical practice. [21, 29, 30] The results of our study are in accord with those reported in the study by Blasi et al., [6] which shows that in Europe the application of PSI and CURB-65 appears well below the threshold recommended by the guidelines. The most encouraging results emerged from university hospitals, where PSI calculation was done in approximately $30 \%$ of cases, compared with $6 \%$ of cases in nonuniversity hospitals, and CURB-65 calculations in around $40 \%$ vs. $14 \%$.

In line with Dambrava et al. [31] we demonstrated an association between adherence to CP in choice of initial antibiotic therapy and reduced length of hospital stay and duration of antibiotic therapy.

The use of NIV in patients with CAP, both in the literature and in our study, remains controversial. [32, 33]

Mortality. In-hospital mortality in our sample was in line with European hospital mortality rates $(0 \%$ to $17.5 \%)$. [6]

In our study, performance of urinary antigen tests was associated with lower mortality, although performance of blood cultures did not yield similar results. This is difficult to compare with existing literature, as such variables are often estimated in combination. For example, Uematsu et al. [11] in 2014 showed that combined performance of blood culture, sputum investigations and urinary antigen tests was associated with a 36\% reduction of 30day mortality $(\mathrm{p}<0.001)$.

Some studies have found lower mortality when antibiotic treatment is adherent to guidelines. [15, 17-19] Menéndez et al. demonstrated that non-adherence was an independent risk factor 
for treatment failure and mortality. [18] Nevertheless, Dambrava et al. [31] found adherence not to be associated with mortality, also consistent with our findings.

Clinical outcomes. In our study, the average length of hospital stay was in line with the European mean duration documented by Blasi et al. (9.0 days). [6]

\section{Data interpretation}

Overall, results from this study showed that clinical practice in our hospital changed over time. Adherence to $\mathrm{CP}$ in the choice of empirical antibiotic therapy appeared to reduce over time, while main diagnostic and therapeutic interventions (i.e. PSI calculation, execution of urinary antigen tests, administration of oxygen therapy) improved.

These mixed results may explain why in-hospital and 30-day mortality did not significantly change in 2012 compared to 2005. Mortality did not appear to be associated with compliance to $\mathrm{CP}$, except for performance of urinary antigen test, but did show association with patient age and CAP severity. It is possible that impact on mortality due to poor compliance with guideline recommended antibiotic therapy could have been cancelled out by improvements in compliance with other diagnostic and therapeutic interventions.

Reduced adherence to CP empiric antibiotic therapy in 2012 compared to 2005 may be due to lack of dedicated resources available for implementation of the clinical guidelines. Although recently in Italy there have been some efforts to promote implementation of clinical guidelines, [34] the frequency and quality of dissemination is still subject to local initiative. A recent Italian study [35] found that implementation of therapeutic recommendations, including empirical antibiotic therapy, led to significantly better outcomes of CAP and/or return to prior health status, especially in more severe cases of CAP.

\section{Limitations of our study}

Our study limitations primarily stem from the observational study design, although we have accounted for the most common potential confounders of the association between adherence to CAP CP and clinical outcomes. As in any retrospective study, missing data can influence the effect size of associations as well as their precision. Particularly, the data relating to administration of antibiotics within 4 hours from access to A\&E and data about pneumococcal and influenza vaccinations were only partially complete, and such variables could not be accounted for in the analyses. However, most variables were carefully recorded and could be included in the analyses.

In 2005 the majority $(62.96 \%)$ of CAP patients were treated in the hospital's respiratory ward, while in 2012, due to a hospital-wide reorganisation, all CAP patients were treated in nonrespiratory wards. This could have potentially influenced both adherence to CAP CP and the clinical outcomes assessed in our study. However, we have accounted for this potential confounding effect by including ward type in the regression model.

\section{CONCLUSION}

Adherence to CAP guidelines can progressively change over time, suggesting the need for continual efforts to promote compliance with clinical guidelines.

In our study, mortality was not associated with compliance to the clinical pathway, but appeared positively associated with patient age and CAP severity, and negatively associated with performance of urinary antigen tests. Adherence to $\mathrm{CP}$ empirical antibiotic treatment was associated with lower length of hospital stay and shorter duration of antibiotic therapy, suggesting overall improvement in clinical care with adherence to the CP. Ongoing and 
comprehensive approaches to favour guidelines implementation may result in improvement of the overall management of CAP and help reduce burden on hospital services.

\section{ACKNOWLEDGEMENTS}

Design of the study: PEB, EC, FP; acquisition of data: EC, EA; interpretation of data: PEB, EC, EA, FP; drafting of the manuscript: PEB, EC, EA, FP; critical revision of the manuscript: PEB, EA, FF, FH.

Conflict of Interest: The authors declare that they have no conflict of interest.

\section{REFERENCES}

1. Blasi F, Mantero M, Santus P, et al. Understanding the burden of pneumococcal disease in adults. Clin Microbiol Infect 2012;18:7-14

2. Ramirez JA, Anzueto AR. Changing needs of community-acquired pneumonia. J Antimicrob Chemother 2011;66:3-9

3. Welte T, Torres A, Nathwani D. Clinical and economic burden of communityacquired pneumonia among adults in Europe. Thorax 2012;67:71-9

4. Bauer TT, Welte T, Ernen C, et al. Cost analyses of community-acquired pneumonia from the hospital perspective. Chest 2005;128:2238-46

5. Millett ERC, De Stavola BL, Quint JK, et al. Time trends and risk factors for hospitalisation after community-acquired pneumonia in older adults in England. Thorax 2014;69:A10

6. Blasi F, Garau J, Medina J, et al. Current management of patients hospitalized with community-acquired pneumonia across Europe: outcomes from REACH. Respir Res 2013;14:44

7. Capelastegui A, Espana PP, Quintana JM, et al. Improvement of process-of-care and outcomes after implementing a guideline for the management of community-acquired pneumonia: A controlled before-and-after design study. Clin Infect Dis 2004;39:95563

8. Marrie TJ, Lau CY, Wheeler SL, et al. A controlled trial of a critical pathway for treatment of community-acquired pneumonia. CAPITAL Study Investigators. Community-Acquired Pneumonia Intervention Trial Assessing Levofloxacin. JAMA 2000;283:749-55

9. Blasi F, Iori I, Bulfoni A, et al. Can CAP guideline adherence improve patient outcome in internal medicine departments? Eur Resp J 2008;32:902-10

10. McCabe C, Kirchner C, Zhang H, et al. Guideline-Concordant Therapy and Reduced Mortality and Length of Stay in Adults With Community-Acquired Pneumonia. Arch Intern Med 2009;169:1525-31

11. Uematsu $\mathrm{H}$, Hashimoto $\mathrm{H}$, Iwamoto $\mathrm{T}$, et al. Impact of guideline-concordant microbiological testing on outcomes of pneumonia. Int $\mathrm{J}$ Qual Health Care 2014;26:100-7

12. Frei CR, Restrepo MI, Mortensen EM, et al. Impact of guideline-concordant empiric antibiotic therapy in community-acquired pneumonia. Am J Med 2006;119:865-71

13. Menéndez R, Reyes S, Martinez R, et al. Economic evaluation of adherence to treatment guidelines in nonintensive care pneumonia. Eur Respir J 2007;29:751-6

14. Ostermann H, Garau J, Medina J, et al. Resource use by patients hospitalized with community-acquired pneumonia in Europe: analysis of the REACH study. BMC 
Pulm Med 2014;14:36

15. Menéndez R, Ferrando D, Vallés JM, et al. Influence of Deviation From Guidelines on the Outcome of Community-Acquired Pneumonia. Chest 2002;122:612-7

16. Dean NC, Bateman KA, Donnelly SM, et al. Improved clinical outcomes with utilization of a community-acquired pneumonia guideline. Chest 2006;130:794-9

17. Mortensen EM, Restrepo M, Anzueto A, et al. Effects of guideline-concordant antimicrobial therapy on mortality among patients with community-acquired pneumonia. Am J Med 2004;117:726-31

18. Menéndez R, Torres A, Zalacaín R, et al. Guidelines for the treatment of communityacquired pneumonia: predictors of adherence and outcome. Am J Respir Crit Care Med 2005;172:757-62

19. Dean NC, Silver MP, Bateman KA, et al. Decreased mortality after implementation of a treatment guideline for community-acquired pneumonia. Am J Med. 2001;110:4517

20. Cabana MD, Rand CS, Powe NR, et al. Why don't physicians follow clinical practice guidelines? A framework for improvement. JAMA 1999;282:1458-65

21. Woodhead M, Blasi F, Ewig S, et al. Guidelines for the management of adult lower respiratory tract infections - Full version. Clin Microbiol Infect 2011;17:E1-59

22. Niederman MS, Mandell LA, Anzueto A, et al. American Thoracic Society. Guidelines for the management of adults with Community-Acquired Pneumonia. Am J Respir Crit Care Med;2001:1730-54

23. British Thoracic Society Standards of Care Committee. BTS guidelines for the management of community acquired pneumonia in adults. Thorax 2001;56:1-58

24. von Elm E, Altman DG, Egger M, et al. The Strengthening the Reporting of Observational Studies in Epidemiology (STROBE) Statement: Guidelines for reporting observational studies. Int J Surg 2014;14:S1743-9191

25. Ospedale Maggiore della Carità. Standards of service of the Trauma and Orthopaedics ward. 2014. Available at: http://www.maggioreosp.novara.it/site/home/attivitaassistenziale/reparti-e-servizi-sanitari/elenco-delle-strutturesanitarie/documento8021635.html

26. Italian National Institute of Statistics (ISTAT). "Geodemo" demographic data. 2013. Available at: http://demo.istat.it/pop2013/index.html. Accessed August 30, 2014

27. Ewig S. The pneumonia triad. In: Chalmers JD, Pletz MW, Aliberti S, eds. Community-Acquired Pneumonia. Eur Respir Monogr 2014;63:13-24

28. Mandell LA, Wunderink RG, Anzueto A, et al. Infectious Diseases Society of America/American Thoracic Society consensus guidelines on the management of community-acquired pneumonia in adults. Clin Infect Dis 2007;44:S27-72

29. Lim WS, Baudouin SV, George RC, et al. British Thoracic Society guidelines for the management of community acquired pneumonia in adults: update 2009. Thorax 2009;64:1-55

30. Bonaiti G, Aliberti S, Suigo G, et al. When do we need to hospitalize a patient with community-acquired pneumonia? Rassegna di Patologia dell'Apparato Respiratorio 2013;28:189-95

31. Dambrava PG, Torres A, Valles X, et al. Adherence to guidelines' empirical antibiotic recommendations and community-acquired pneumonia outcome. Eur Respir J 2008;32:892-901

32. Carrillo A, Gonzalez-Diaz G, Ferrer M, et al. Non-invasive ventilation in communityacquired pneumonia and severe acute respiratory failure. Intensive Care Med 2012;38:458-66

33. Ferrer M, Cosentini R, Nava S. The use of non-invasive ventilation during acute 
respiratory failure due to pneumonia. Eur J Intern Med 2012;23;420-8

34. Conferenza Stato Regioni. CSR del 5 agosto 2014: definizione degli standard qualitativi, strutturali, tecnologici e quantitativi relativi all'assistenza ospedaliera.

35. Iori I, Gussoni G, Blasi F, et al. Guidelines and management of hospitalized patients with community-acquired pneumonia: the Italian experience of the FASTCAP study. 


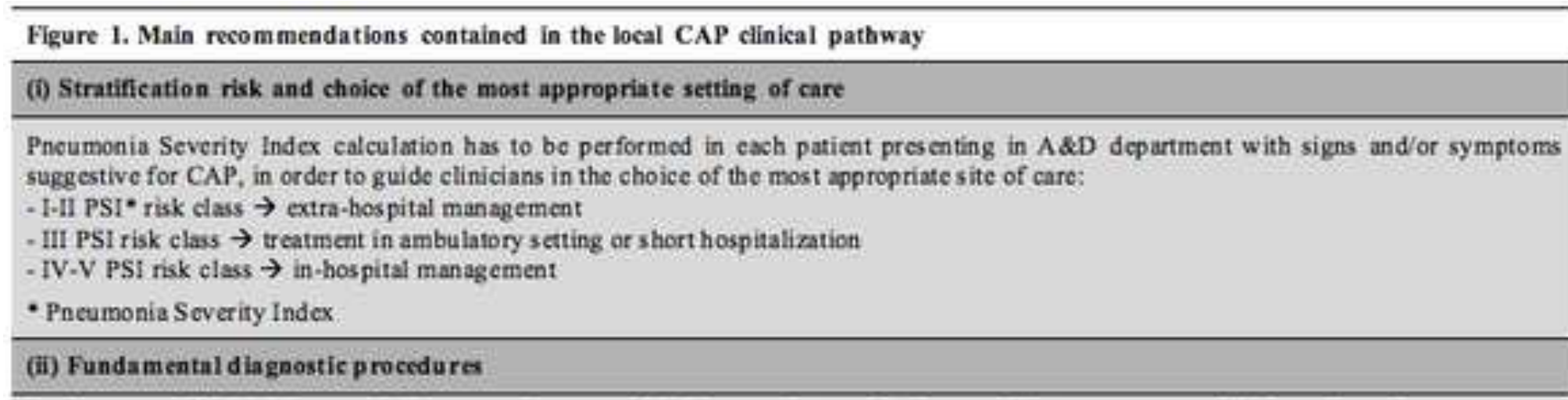

Performance of blood culture and urinary antigen tests for S. Pneumoniae and L. Pneumophila are recommended for each patient.

(iii) Empirical astiblotic therapy

Firs dose of empirical antibiotic therapy has to be performed within 4 hours from access to A\&D department.

The choice of the most appropriate antibiotic scheme might consider clinical aspects defining the following six category of patients

\section{Category $1:$ inpatient, non-Intensive Care Unit treatment}

- Macrolide

- Fluoroquinolone

$-\beta$-lactam + fluoroquinolone or macrolide

\section{Category 2: patient with suspected aspiration from home}

- Amoxicillin-clavulanate

- Levofloxacin + clindamicyn/metronidazole

Category 3: patient with suspected aspiration from nursing home or in Intensive Care Unit treatment

- $\beta$-lactam (ampicillin/sulbactam or high dose ampicillin or other active $\beta$-lactams) + clindamicyn/metronidazole

\section{Category 4: patient with suspected P. aeruginosa infection}

-Anti-Pseudomons $\beta$-lactam (piperacillin-tazobactam, cefepime, imipenem o meropenem) + fluoroquinolone \pm aminoglycoside - Anth.Pscudomonas B-lactam + minoglycoside + macrolide

\section{Category 5: inpatient, Intensive Care Unit treatment without risk for P. aeruginosa infection}

$-\beta$-lectam + fluoroquinolone

- Fluoroquinolone $=$ clindamiein

\section{Category 6: inpatient, Intensive Care Unit treatment with risk for $\mathbf{P}$. aeruginosa infection}

-Anth-Pseuomonas $\beta$-lactam (piperaeillin-tazobactam, cefepime, imipenem o meropenem) + fuoroquinolone

- Aminoglycoside + fluoroquinolone or macrolide

- Aztreonam + fluoroquinolone \pm aminogly coside 


\section{Figure 2. Variables in detail}

(i) personal details and medical history

admission year, admission ward, sex, age, residence at home or nursing home, smoke, anti-Pneumococcal and anti influenza vaccination

(ii) comorbidities

(iii) radiological characteristics of pneumonia

monolateral or bilateral infiltrates in chest $\mathrm{x}$-ray

(iv) presence of pleural effusion or empyema

(v) presence of criteria for severe CAP, as identified by American Thoracic Society [27]

(vi) risk stratification through Pneumonia Severity Index

(vii) diagnostic interventions

procedures aimed to obtain an etiological diagnosis (such as blood culture, urinary antigen tests for S. pneumoniae and L. pneumophila, sputum Gram stain and culture, lung brushing or biopsy, bronchoalveolar lavage, bronchial lavage, quantitative endotracheal as pirate, other) and to manage pleural effusion and/or empyema (such as thoracentesis, chest drainage, video-ass isted thoracic surgery

\section{(viii) in-hospital therapeutic interventions}

timing of administration of the first antibiotic dose, adherence of initial empiric antibiotic therapy to the recommendations of our local clinical pathway (see Figure 1), performance of switch therapy from intravenous to oral therapy, antibiotic therapy duration, oxygen and low molecular weight heparin administration

\section{(ix) in-hospital clinical evolution}

need for invasive and non invasive mechanical ventilation (Mechanical Ventilation), transfer to Intensive Care Unit (ICU)

(x) clinical outcomes

length of hospital stay, in-hospital mortality, 30-day mortality 
Selection on the basis of the discharge diagnosis (i.e. ICD-9 codes reported on HDR)

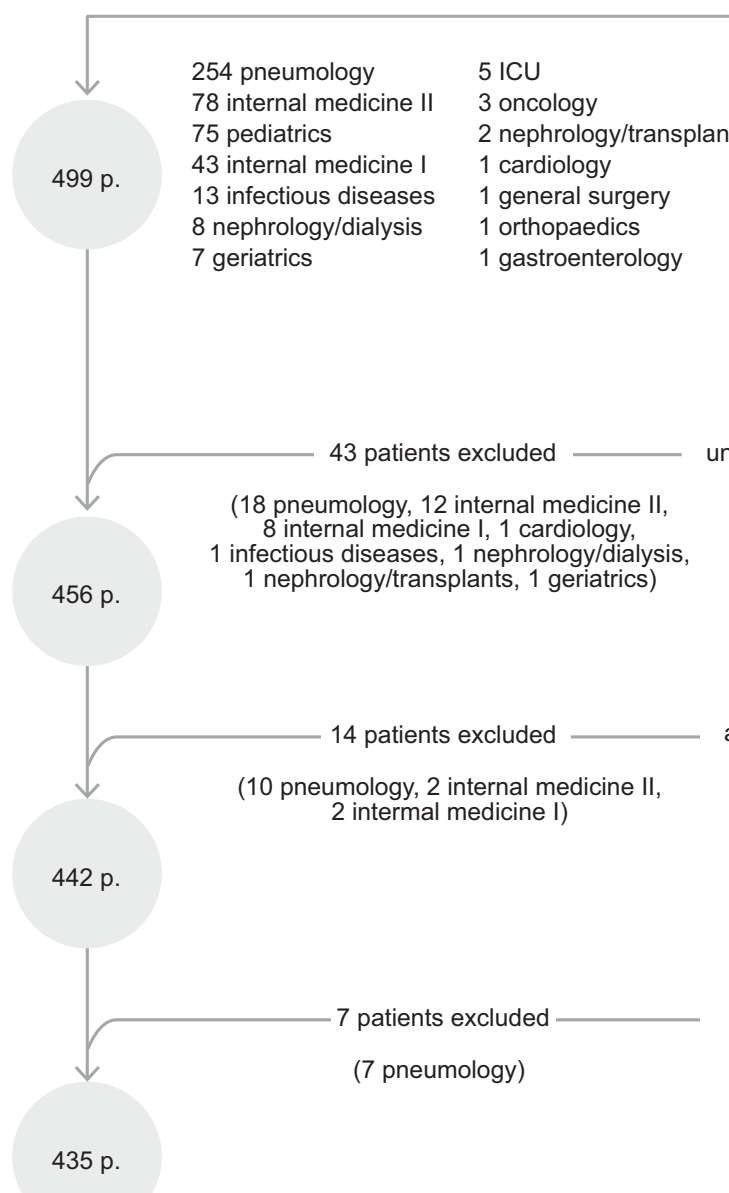

\section{$1102 p$.}

78 internal medicine II 3 oncology

75 pediatrics 2 nephrology/transplants

13 infectious diseases 1 general surgery

8 nephrology/dialysis 1 orthopaedics

gastroenterology

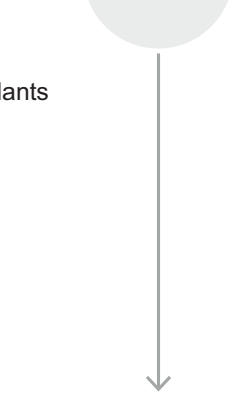

210 internal medicine II 4 nursing home

161 internal medicine I $4 \mathrm{ICU}$

58 infectious diseases 3 nephrology/transplants

34 internal medicine III 2 cardiology

33 pneumology 2 emergency department

28 pediatrics 1 cardiosurgery

19 thoracic surgery 1 general surgery

17 oncology 1 neurology

15 hematoloy 1 gastroenterology

8 nephrology/dialysis 1 radiotherapy

unavaliable clinical records

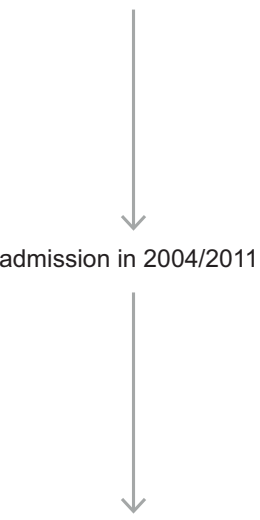

day hospital admission

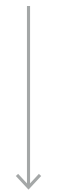

unrespected inclusion criteria (including unconfirmed CAP

diagnosis)

(75 pediatrics, 50 pneumology, 8 internal medicine II, 7 internal medicine I, 4 thoracic surgery, 2 ICU,

$283 \mathrm{p} . \quad 2$ geriatrics, 1 general surgery, 1 infectious diseases, 1 orthopaedics, 1 gastroenterology)

40 patients excluded

(5 chemiotherapy/immunosuppression:

3 oncology, 1 nephrology/transplants, 1 infectious diseases;

35 recent admission: 16 pneumology,

243 p. $\quad 7$ internal medicine II, 6 internal medicine I,

4 nephrology/dialysis, 2 infectious diseases)

256 patients excluded

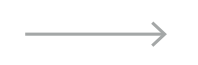

patients excluded

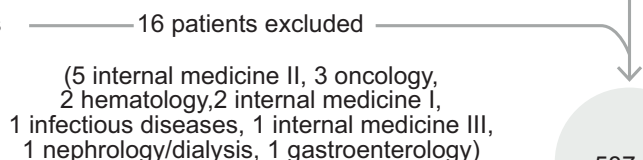

587 p. 
Table 1. Socio-demographic and clinical characteristics of the sample

\begin{tabular}{|c|c|c|c|c|}
\hline & 2005 & 2012 & total & $p$-value \\
\hline Age in years, mean (SD) & $69.03(20.69)$ & $75.36(15.58)$ & $72.64(18.22)$ & $<0.0001$ \\
\hline $\begin{array}{c}\text { Sex, } \%(\mathrm{n}) \\
\text { male } \\
\text { female }\end{array}$ & $\begin{array}{l}57.61(140) \\
42.39(103)\end{array}$ & $\begin{array}{l}56.39(181) \\
43.61(140)\end{array}$ & $\begin{array}{l}56.91(321) \\
43.09(243)\end{array}$ & 0.771 \\
\hline $\begin{array}{l}\text { Admission from, } \% \text { (n) } \\
\text { home } \\
\text { nursing home }\end{array}$ & $\begin{array}{c}90.95(221) \\
9.05(22)\end{array}$ & $\begin{array}{c}85.98(276) \\
14.02(45)\end{array}$ & $\begin{array}{c}88.12(497) \\
11.88(67)\end{array}$ & 0.071 \\
\hline $\begin{array}{l}\text { Smoking, \% (n) } \\
\quad \text { smokers } \\
\text { never smokers } \\
\text { ex smokers } \\
\text { not assessed }\end{array}$ & $\begin{array}{l}20.58(50) \\
38.68(94) \\
20.16(49) \\
20.58(50)\end{array}$ & $\begin{array}{c}16.20(52) \\
35.83(115) \\
29.28(94) \\
18.69(60)\end{array}$ & $\begin{array}{l}18.09(102) \\
37.06(209) \\
25.35(143) \\
19.50(110)\end{array}$ & 0.087 \\
\hline $\begin{array}{l}\text { Comorbidity, } \%(\mathrm{n}) \\
\text { systemic hypertension } \\
\text { ischemic cardiopathy } \\
\text { heart failure } \\
\text { cerebrovascular disease } \\
\text { diabetes mellitus } \\
\text { malignancy } \\
\text { chronic liver disease } \\
\text { chronic kidney disease } \\
\text { chronic lung disease } \\
\text { non-neoplastic haematological disease* } \\
\text { diseases non otherwise classified } \\
\geq \mathbf{5} \text { comorbidities }\end{array}$ & $\begin{array}{c}\mathbf{3 6 . 2 1}(\mathbf{8 8}) \\
17.28(42) \\
19.34(47) \\
\mathbf{1 8 . 1 1}(\mathbf{4 4 )} \\
16.05(39) \\
\mathbf{1 1 . 5 2}(\mathbf{2 8 )} \\
9.05(22) \\
13.99(34) \\
24.69(60) \\
\mathbf{2 . 0 6}(\mathbf{5}) \\
\mathbf{6 5 . 0 2}(\mathbf{1 5 8}) \\
\mathbf{9 . 0 5}(\mathbf{2 2})\end{array}$ & $\begin{array}{c}\mathbf{5 1 . 4 0}(\mathbf{1 6 5}) \\
19.00(61) \\
25.55(82) \\
\mathbf{3 5 . 2 0}(\mathbf{1 1 3}) \\
21.18(68) \\
\mathbf{2 4 . 9 2 ( 8 0 )} \\
11.53(37) \\
12.15(39) \\
28.66(92) \\
\mathbf{6 . 5 4}(\mathbf{2 1}) \\
\mathbf{7 6 . 0 1 ( 2 4 4 )} \\
\mathbf{1 9 . 3 1 ( 6 2 )}\end{array}$ & $\begin{array}{l}\mathbf{4 4 . 8 6}(\mathbf{2 5 3}) \\
18.26(103) \\
22.87(129) \\
\mathbf{2 7 . 8 4}(\mathbf{1 5 7}) \\
18.97(107) \\
\mathbf{1 9 . 1 5 ( 1 0 8 )} \\
10.46(59) \\
12.94(73) \\
26.95(152) \\
\mathbf{4 . 6 1 ( 2 6 )} \\
\mathbf{7 1 . 2 8 ( 4 0 2 )} \\
\mathbf{1 4 . 8 9}(\mathbf{8 4 )}\end{array}$ & $\begin{array}{c}<\mathbf{0 . 0 0 1} \\
0.601 \\
0.082 \\
<\mathbf{0 . 0 0 1} \\
0.124 \\
<\mathbf{0 . 0 0 1} \\
0.342 \\
0.519 \\
0.293 \\
\mathbf{0 . 0 1 2} \\
\mathbf{0 . 0 0 4} \\
\mathbf{0 . 0 0 1}\end{array}$ \\
\hline
\end{tabular}

Note: in bold are the variables for which there is evidence at the 5\% level of a difference between 2005 and 2012

* non-immunosuppressive non-neoplastic haematological disease such as anemia and myelodysplastic diseases 
Table 2. Radiological aspects of pneumonia and criteria for severe CAP

\begin{tabular}{|c|c|c|c|c|}
\hline & 2005 & 2012 & total & $p$-value \\
\hline \multicolumn{5}{|l|}{ Radiological aspect, \% (n) } \\
\hline XR monolateral infiltrates & $79.83(190)$ & $80.70(255)$ & $80.32(445)$ & \multirow{2}{*}{0.800} \\
\hline XR bilateral infiltrates & $20.17(48)$ & $19.30(61)$ & $19.68(109)$ & \\
\hline pleuric effusion & $31.69(77)$ & $30.22(97)$ & $30.85(174)$ & 0.708 \\
\hline pleuric empyema & $3.29(8)$ & $3.12(10)$ & $3.19(18)$ & 0.906 \\
\hline \multicolumn{5}{|l|}{ Criteria for severe CAP, \% (n) } \\
\hline invasive mechanical ventilation & $1.65(4)$ & $1.25(4)$ & $1.42(8)$ & 0.691 \\
\hline septic shock & $2.47(6)$ & $2.18(7)$ & $2.30(13)$ & 0.821 \\
\hline respiratory rate $>\mathbf{3 0}$ breaths/minute & $7.82(19)$ & $0.93(3)$ & $3.90(22)$ & $<0.001$ \\
\hline $\mathrm{paO}_{2} / \mathrm{FiO}_{2}<250$ & $0.41(1)$ & $0.62(2)$ & $0.53(3)$ & 0.732 \\
\hline multilobar infiltrates & $25.51(62)$ & $24.30(78)$ & $24.82(140)$ & 0.741 \\
\hline confusion/disorientation & $20.16(49)$ & $19.00(61)$ & $19.50(110)$ & 0.730 \\
\hline uremia (Blood Urea Nitrogen > 20 mg/dl) & $26.75(65)$ & $3.12(10)$ & $13.30(75)$ & $<0.001$ \\
\hline leukopenia (WBC < 4000/mm3) & $6.17(15)$ & $4.36(14)$ & $5.14(29)$ & 0.335 \\
\hline thrombocytopenia (platelets $<100000 / \mathrm{mm} 3$ ) & $8.64(21)$ & $6.54(21)$ & $7.45(42)$ & 0.347 \\
\hline hypothermia (body temperature $<36^{\circ} \mathrm{C}$ ) & 0 & 0 & 0 & - \\
\hline systolic blood pressure $<90 \mathrm{mmHg}$ & $2.47(6)$ & $4.05(13)$ & $3.37(19)$ & 0.303 \\
\hline severe CAP as defined by ATS*, \% (n) & $10.70(26)$ & $5.61(18)$ & $7.80(44)$ & 0.026 \\
\hline at least 1 major criteria & $3.29(8)$ & $3.12(10)$ & $3.19(18)$ & 0.906 \\
\hline at least 2 of the first 3 minor criteria & $1.23(3)$ & $2.18(7)$ & $1.77(10)$ & 0.399 \\
\hline at least 3 minor criteria & $9.05(22)$ & $2.18(7)$ & $5.14(29)$ & $<0.001$ \\
\hline
\end{tabular}

Note: in bold are the variables for which there is evidence at the 5\% level of a difference between 2005 and 2012

*American Thoracic Society 
Table 3. Adherence to diagnostic procedures and its main findings

\begin{tabular}{|c|c|c|c|c|}
\hline & 2005 & 2012 & total & $p$-value \\
\hline PSI (Pneumonia Severity Index) calculation, \% (n) & $17.70(43)$ & $25.23(81)$ & $21.99(124)$ & 0.032 \\
\hline I class & $4.65(2)$ & $12.35(10)$ & $9.68(12)$ & \\
\hline II class & $4.65(2)$ & $8.64(7)$ & $7.26(9)$ & \\
\hline III class & $13.95(6)$ & $20.99(17)$ & $18.55(23)$ & 0.146 \\
\hline IV class & $60.47(26)$ & $37.04(30)$ & $45.16(56)$ & \\
\hline $\mathrm{V}$ class & $16.28(7)$ & $20.99(17)$ & $19.35(24)$ & \\
\hline History investigation about vaccinations, \% (n) & $0.41(1)$ & 0 & $0.01(1)$ & 0.250 \\
\hline \multicolumn{5}{|l|}{ Procedures for etiological diagnosis, \% (n) } \\
\hline blood culture & $60.91(148)$ & $64.17(206)$ & $62.77(354)$ & 0.426 \\
\hline positive & $24.32(36)$ & $9.71(20)$ & $15.82(56)$ & $<0.001$ \\
\hline S. pneumoniae & $2.78(1)$ & $35.00(7)$ & $14.29(8)$ & \multirow{6}{*}{0.020} \\
\hline S. aureus & $2.78(1)$ & $10.00(2)$ & $5.36(3)$ & \\
\hline coagulase-negative Staphylococci & $66.67(24)$ & $40.00(8)$ & $57.14(32)$ & \\
\hline Pseudomonas vesicularis & $2.78(1)$ & $\mathbf{0}$ & $1.79(1)$ & \\
\hline Enterobacteriaceae & $8.33(3)$ & $5.00(1)$ & $7.14(4)$ & \\
\hline Saprophytic & $16.67(6)$ & $10.00(2)$ & $14.29(8)$ & \\
\hline urinary antigen test for $S$. pneumoniae & $22.63(55)$ & $56.39(181)$ & $41.84(236)$ & $<0.001$ \\
\hline positive & $3.64(2)$ & $6.08(11)$ & $5.51(13)$ & 0.487 \\
\hline urinary antigen test for $L$. pneumophila & $48.56(118)$ & $57.63(185)$ & $53.72(303)$ & 0.032 \\
\hline positive & $1.69(2)$ & $4.32(8)$ & $3.30(10)$ & 0.212 \\
\hline sputum Gram stain & $19.75(48)$ & $0.93(3)$ & $9.04(51)$ & $<0.001$ \\
\hline sputum culture & $29.63(72)$ & $19.31(62)$ & $23.76(134)$ & 0.004 \\
\hline lung brushing or biopsy & $4.94(12)$ & $1.25(4)$ & $2.84(16)$ & 0.009 \\
\hline bronchoalveolar lavage (BAL) & $4.94(12)$ & $8.72(28)$ & $7.09(40)$ & 0.083 \\
\hline bronchial lavage $(\mathrm{BL})$ & $10.70(26)$ & $0.31(1)$ & $4.79(27)$ & $<0.001$ \\
\hline quantitative endotracheal aspirate & $1.23(3)$ & $2.49(8)$ & $1.95(11)$ & 0.285 \\
\hline other & $61.73(150)$ & $45.17(145)$ & $52.30(295)$ & $<0.001$ \\
\hline \multicolumn{5}{|l|}{ Procedures for pleural effusion/empyema, \% (n) } \\
\hline thoracentesis & $22.35(19)$ & $14.95(16)$ & $18.23(35)$ & 0.187 \\
\hline chest drainage & $9.41(8)$ & $9.35(10)$ & $9.38(18)$ & 0.988 \\
\hline video-assisted thoracic surgery & $37.50(3)$ & $10.00(1)$ & $22.22(4)$ & 0.163 \\
\hline
\end{tabular}

Note: in bold are the variables for which there is evidence at the 5\% level of a difference between 2005 and 2012 
Table 4. Adherence to therapeutic interventions

\begin{tabular}{|c|c|c|c|c|}
\hline & 2005 & 2012 & total & p-value \\
\hline \multicolumn{5}{|l|}{$\begin{array}{l}\text { Distribution of patients within clinical categories } \\
\text { identified by guidelines } \%(\mathrm{n})\end{array}$} \\
\hline 1) inpatient, non-ICU* treatment & $96.71(235)$ & $96.26(309)$ & $96.45(544)$ & \\
\hline 2) patient with suspected aspiration from home & $1.23(3)$ & $0.62(2)$ & $0.89(5)$ & \\
\hline $\begin{array}{l}\text { 3) patient with suspected aspiration from nursing home or in } \\
\text { ICU treatment }\end{array}$ & $0.41(1)$ & $1.56(5)$ & $1.06(6)$ & \\
\hline 4) patient with suspected P. aeruginosa infection & $0.41(1)$ & $1.25(4)$ & $0.89(5)$ & 0.151 \\
\hline $\begin{array}{l}\text { 5) inpatient, ICU treatment without risk for P. aeruginosa } \\
\text { infection }\end{array}$ & $1.23(3)$ & - & $0.53(3)$ & \\
\hline $\begin{array}{l}\text { 6) inpatient, ICU treatment with risk for P. aeruginosa } \\
\text { infection }\end{array}$ & - & $0.31(1)$ & $0.18(1)$ & \\
\hline \multicolumn{5}{|l|}{ Antibiotic therapy adherent to guidelines, $\%$ (n) } \\
\hline in patients of category 1 & $70.26(163)$ & $58.58(181)$ & $63.59(344)$ & 0.005 \\
\hline in patients of category 2 & $33.33(1)$ & 0 & $20.00(1)$ & 0.361 \\
\hline in patients of category 3 & 0 & 0 & 0 & - \\
\hline in patients of category 4 & 0 & $25.00(1)$ & $20.00(1)$ & 0.576 \\
\hline in patients of category 5 & $33.33(1)$ & - & $33.33(1)$ & - \\
\hline in patients of category 6 & - & 0 & 0 & - \\
\hline $\begin{array}{l}\text { Administration of the first antibiotic dose, } \%(n) \\
\text { within } 4 \text { hours from A\&E presentation } \\
\text { within } 4 \text { hours from admission to ward }\end{array}$ & $\begin{array}{l}96.00(24) \\
54.92(106)\end{array}$ & $\begin{array}{l}99.59(243) \\
69.66(202)\end{array}$ & $\begin{array}{l}99.26(267) \\
63.77(308)\end{array}$ & $\begin{array}{c}0.047 \\
<0.001\end{array}$ \\
\hline Switch therapy, \% (n) & $23.31(55)$ & $30.94(99)$ & $27.70(154)$ & 0.047 \\
\hline Intra-hospital antibiotic therapy duration in days, mean (SD) & $10.71(7.17)$ & $10.56(6.39)$ & $10.63(6.73)$ & 0.7925 \\
\hline Post-discharge prescription duration in days, mean (SD) & $6.20(2.52)$ & $4.79(2.33)$ & $5.39(2.33)$ & $<0.0001$ \\
\hline Antibiotic therapy total duration in days, mean (SD) & $13.66(8.18)$ & $12.78(6.65)$ & $13.14(7.33)$ & 0.1825 \\
\hline $\begin{array}{l}\text { Oxygen administration, } \% \text { (n) } \\
\text { MV (mechanical ventilation), \% (n) }\end{array}$ & $\begin{array}{l}58.85(143) \\
2.06(5)\end{array}$ & $\begin{array}{l}75.70(243) \\
9.35(30)\end{array}$ & $\begin{array}{l}68.44(386) \\
6.21(35)\end{array}$ & $\begin{array}{l}<0.001 \\
<0.001\end{array}$ \\
\hline Administration of low molecular weight heparin, $\%$ (n) & $53.09(129)$ & $58.88(189)$ & $56.38(318)$ & 0.170 \\
\hline
\end{tabular}

Note: in bold are the variables for which there is evidence at the 5\% level of a difference between 2005 and 2012

* Intensive Care Unit 
Table 5. Multivariable regression analyses for in-hospital and 30-day mortality, length of hospital stay and duration of antibiotic therapy

\begin{tabular}{|c|c|c|c|c|c|c|c|c|}
\hline & \multicolumn{2}{|c|}{ in-hospital mortality } & \multicolumn{2}{|c|}{ 30-day mortality } & \multicolumn{2}{|c|}{ length of hospital stay } & \multicolumn{2}{|c|}{$\begin{array}{c}\text { duration of antibiotic } \\
\text { therapy }\end{array}$} \\
\hline & \multicolumn{2}{|r|}{$\mathrm{N}=561$} & \multicolumn{2}{|c|}{$\mathrm{N}=495$} & \multicolumn{2}{|r|}{$\mathrm{N}=561$} & \multicolumn{2}{|r|}{$\mathrm{N}=505$} \\
\hline & OR & $95 \% \mathrm{CI}$ & OR & $95 \% \mathrm{CI}$ & MD & $95 \% \mathrm{CI}$ & MD & $95 \% \mathrm{CI}$ \\
\hline \multicolumn{9}{|c|}{ Socio-demographic characteristics and other potential confounders } \\
\hline Age in years (continuous) & 1.081 & 1.049,1.114 & 1.105 & $1.068,1.143$ & 0.052 & $0.016,0.089$ & -0.003 & $-0.038,0.033$ \\
\hline $\begin{array}{l}\text { Male gender (vs. female } \\
\text { gender) }\end{array}$ & 0.803 & $0.454,1.419$ & 0.628 & $0.348,1.133$ & 0.850 & $-0.424,2.124$ & 0.411 & $-0.792,1.615$ \\
\hline $\begin{array}{l}\text { Admission from nursing } \\
\text { home (vs. from own home) }\end{array}$ & 1.118 & $0.544,2.300$ & 1.409 & $0.648,3.063$ & -3.222 & $-5.232,-1.213$ & -1.770 & $-3.619,0.079$ \\
\hline $\begin{array}{l}\text { Five or more comorbidities } \\
\text { (vs. less than five) }\end{array}$ & 1.601 & $0.827,3.102$ & 1.243 & $0.627,2.464$ & 1.150 & $-0.621,2.921$ & 1.421 & $-0.233,3.075$ \\
\hline $\begin{array}{l}\text { ATS criteria for CAP } \\
\text { severity (continuous) }\end{array}$ & 1.743 & $1.383,2.196$ & 1.868 & $1.462,2.385$ & -0.287 & $-0.903,0.329$ & -1.111 & $-1.694,-0.527$ \\
\hline $\begin{array}{l}\text { Admission in } 2012 \text { (vs. } \\
\text { admission in 2015) }\end{array}$ & 1.081 & $0.491,2.377$ & 0.863 & $0.387,1.923$ & 1.297 & $-0.723,3.318$ & 0.659 & $-1.224,2.541$ \\
\hline $\begin{array}{l}\text { Stay in a respiratory ward } \\
\text { (vs. stay in a non-respiratory } \\
\text { ward) }\end{array}$ & 0.847 & $0.351,2.042$ & 0.750 & $0.304,1.851$ & 4.225 & $2.152,6.299$ & 4.363 & $2.377,6.349$ \\
\hline Mechanical Ventilation & 0.968 & $0.289,3.243$ & 1.102 & $0.340,3.575$ & 4.638 & $1.688,7.587$ & 5.017 & 2.115,7.919 \\
\hline \multicolumn{9}{|c|}{ Adherence to diagnostic procedures } \\
\hline Blood culture & 0.677 & $0.377,1.213$ & 0.600 & $0.328,1.097$ & 2.631 & $1.259,4.003$ & 2.728 & $1.417,4.039$ \\
\hline Urinary Antigen tests & 0.427 & $0.215,0.850$ & 0.341 & $0.170,0.685$ & 0.033 & $-1.407,1.473$ & -0.164 & $-1.535,1.207$ \\
\hline \multicolumn{9}{|c|}{ Adherence to therapeutic interventions } \\
\hline $\begin{array}{l}\text { Antibiotic therapy adherent } \\
\text { to clinical pathway }\end{array}$ & 0.830 & $0.468,1.471$ & 0.957 & $0.526,1.738$ & -2.868 & $-4.186,-1.550$ & -2.004 & $-3.265,-0.743$ \\
\hline Treatment in ICU & 0.452 & $0.073,2.817$ & 0.305 & $0.047,1.983$ & 8.409 & 3.547,13.272 & 6.237 & $1.170,11.303$ \\
\hline
\end{tabular}

Note: in bold are the variables for which there is evidence at the 5\% level of a difference between 2005 and 2012 